\title{
Clinical significance of lymph node size in locally advanced cervical cancer treated with concurrent chemoradiotherapy
}

\author{
Jinju Oh ${ }^{1}$, Ki Ho Seol ${ }^{2}$, Youn Seok Choi ${ }^{1}$, Jeong Won Lee ${ }^{2}$, Jin Young Bae ${ }^{1}$ \\ ${ }^{1}$ Department of Obstetrics and Gynecology, Catholic University of Daegu School of Medicine, Daegu, Korea \\ ${ }^{2}$ Department of Radiation Oncology, Catholic University of Daegu School of Medicine, Daegu, Korea
}

Received: January 7, 2019

Revised: February 14, 2019

Accepted: February 18, 2019

Corresponding author:

Ki Ho Seol

Department of Radiation Oncology, Catholic University of Daegu School of Medicine, 33, Duryugongwon-ro

17-gil, Nam-gu, Daegu 42472,

Korea

Tel: +82-53-650-4788

Fax: +82-53-289-2697

E-mail:khseol@cu.ac.kr
Background: This study aimed to assess the in-field lymph node (LN) failure rate according to $L N$ size and to investigate effect of $L N$ size on the survival outcome of patients with locally advanced cervical carcinoma treated with concurrent chemoradiotherapy (CCRT).

Methods: A total of 310 patients with locally advanced cervical carcinoma treated with CCRT were enrolled in retrospective study. LN status was evaluated by magnetic resonance imaging. All patients received conventional external beam irradiation and high-dose rate brachytherapy, and concurrent cisplatin-based chemotherapy. In-field LN failure rate according to LN size was analyzed.

Results: The median follow-up period was 83 months (range, 3-201 months). In-field LN failure rate in patients with pelvic $L N$ size more than $10 \mathrm{~mm}$ was significantly higher than that in patients with pelvic $L N$ size less than $10 \mathrm{~mm}(p<0.001)$. A similar finding was observed in the infield para-aortic LN (PALN) failure rate $(p=0.024)$. The pelvic and PALN size $(\geq 10 \mathrm{~mm})$ was a significant prognostic factor of overall-survival (OS) and disease-free survival rate in univariate and multivariate analyses. The OS rate was significantly different between groups according to LN size ( $<10 \mathrm{~mm}$ vs. $\geq 10 \mathrm{~mm})$.

Conclusion: A LN of less than $10 \mathrm{~mm}$ in size in an imaging study is controlled by CCRT. On the other hand, in LN of more than $10 \mathrm{~mm}$ in size, the in-field $L N$ failure rate increase and the prognosis deteriorate. Therefore, a more aggressive treatment strategy is needed.

Keywords: Chemoradiotherapy; Lymph node; Uterine cervical neoplasms

\section{Introduction}

Cervical cancer is the fourth most common cancer worldwide with an annual mortality rate of 250,000 in developing countries [1]. Since 1999, five phase III randomized clinical trials reported significant survival advantages for patients who received concurrent chemoradiotherapy (CCRT) compared with those who received radiotherapy alone [2]. CCRT has become the standard treatment for locally advanced cervical cancer [3].

The incidence of lymph node (LN) involvement in locally advanced cervical cancer is $39-44 \%$ and the incidence of paraaortic LN (PALN) involvement is approximately 8-16\% [4-6]. Radiotherapy for metastatic regional LNs is not well established in cervical cancer. Recent study about radiation field failure after definitive CCRT in patients with locally advanced cervical cancer (stage IB-IVA) reported that the estimated 3-year rate of locoregional control was about $89 \%$ [7]. There are variables that affect in-field failure, such as tumor size $(>5 \mathrm{~cm})$, young age ( $<40$ years), non-squamous histology and positive LN [7]. It is assumed that control of metastatic regional LNs will be of

Copyright@ 2019 Yeungnam University College of Medicine

This is an Open Access article distributed under the terms of the Creative Commons Attribution Non-Commercial License (http://creativecommons.org/licenses/by-nc/4.0/) which permits unrestricted non-commercial use, distribution, and reproduction in any medium, provided the original work is properly cited. 
clinical significance in patients who do not have local failure. The staging of cervical cancer follows the International Federation of Gynecology and Obstetrics (FIGO) stage system based on clinical staging. Although LN metastasis serves an important role in prognosis, LN status is not included in the staging [8]. In general, the larger the size of the primary tumor, the greater the dose required for achieving tumor control [9-11]. Similarly, the dose required to achieve local control of a metastatic LN increases with the size of the metastasis. The larger the size of the LN, the poorer the prognosis and local control rate. In many institutions, external beam boost irradiation has been used empirically for large metastatic LNs. However, there is limited clinical data for external beam irradiation to metastatic LNs [12-15], and the association between the size of the metastatic LN and LN control in CCRT remains to be fully elucidated. Previous studies included mixed groups comprising patients treated with CCRT or radiotherapy alone, which is inadequate to validate the effect of CCRT. Therefore, we aimed to assess the in-field LN failure rate according to $\mathrm{LN}$ size and to investigate the effect of LN size on the survival outcome in patients with cervical cancer treated with CCRT.

\section{Materials and methods}

\section{Patients}

This retrospective study was approved by the Institutional Review Board in Daegu Catholic University Medical Center (IRB No. CR17-045-L). The medical records of 335 patients with cervical cancer treated with CCRT at the Daegu Catholic University Medical Center between 2000 and 2016, were reviewed. The inclusion criteria were as follows: 1) newly diagnosed histologically proven squamous cell carcinoma, adenocarcinoma, or adenosquamous carcinoma of the uterine cervix; 2) treatment using platinum-based CCRT; and 3) clinical and radiologic FIGO stage IB-IVA with no other evidence of distant metastasis. Of 335 patients, 25 patients were excluded for the following reasons: 1 ) surgical intervention prior to CCRT ( $\mathrm{n}=20)$; and 2) incomplete treatment $(\mathrm{n}=5)$. The remaining 310 patients were included in the analysis. LN metastasis was evaluated by magnetic resonance imaging (MRI).

A total of 142 patients (45.8\%) had metastatic pelvic LNs and 17 patients (5.5\%) had metastatic PALNs. To investigate the effect of $\mathrm{LN}$ size on the treatment outcome, the patients were divided based on the LN size regardless of MRI evaluation results: those who had pelvic LN size less than $10 \mathrm{~mm}(\mathrm{n}=196)$, those who had pelvic LN size from $10 \mathrm{~mm}$ to $19.99 \mathrm{~mm}(\mathrm{n}=90)$, and those who had pelvic LN size $20 \mathrm{~mm}$ or more $(\mathrm{n}=24)$. Further, 189 patients had PALN size less than $5 \mathrm{~mm}, 108$ patients had PALN size from $5 \mathrm{~mm}$ to $9.99 \mathrm{~mm}$ and 13 patients had PALN size $10 \mathrm{~mm}$ or more.
Table 1. The characteristics of the enrolled patients

\begin{tabular}{|c|c|}
\hline Variable & Value $(\%)$ \\
\hline Age (yr, mean $\pm S D)$ & $53.0 \pm 11.4$ \\
\hline Pretreatment hemoglobin $(\mathrm{g} / \mathrm{dL}$, mean $\pm \mathrm{SD})$ & $11.7 \pm 1.62$ \\
\hline Pretreatment SCC Ag. level (ng/mL, mean \pm SD) & $9.9 \pm 16.1$ \\
\hline \multicolumn{2}{|l|}{ Pathology } \\
\hline Squamous cell carcinoma & $257(82.9)$ \\
\hline Adenocarcinoma or ASC & $53(17.1)$ \\
\hline \multicolumn{2}{|l|}{ Stage } \\
\hline IB1 & $53(17.1)$ \\
\hline IB2 & $46(14.8)$ \\
\hline$\| \mathrm{A} 1$ & $20(6.5)$ \\
\hline IIA2 & $12(3.9)$ \\
\hline IIB & $122(39.4)$ \\
\hline IIIA & $2(0.6)$ \\
\hline IIIB & $46(14.8)$ \\
\hline IVA & $9(2.9)$ \\
\hline \multicolumn{2}{|l|}{ Differentiation } \\
\hline Well & $2(0.6)$ \\
\hline Moderately & $286(92.3)$ \\
\hline Poorly & $26(8.4)$ \\
\hline \multicolumn{2}{|l|}{ LVI } \\
\hline Absent & $284(91.6)$ \\
\hline Present & $26(8.4)$ \\
\hline Primary tumor size $\left(\mathrm{mm}\right.$, mean $\left._{ \pm} \mathrm{SD}\right)$ & $40.4 \pm 14.5$ \\
\hline Pelvic LN metastasis & $142(45.8)$ \\
\hline Pelvic LN size $(\mathrm{mm}, \mathrm{mean} \mathrm{SD})$ & $8.9 \pm 7.1$ \\
\hline \multicolumn{2}{|l|}{ Pelvic LN size (mm) } \\
\hline$<10$ & $196(63.2)$ \\
\hline 10-19.99 & $90(29.0)$ \\
\hline$\geq 20$ & $24(7.7)$ \\
\hline PALN metastasis & $17(5.5)$ \\
\hline PALN size $\left(m m, m_{e a n} \pm S D\right)$ & $4.8 \pm 2.5$ \\
\hline \multicolumn{2}{|l|}{ PALN size $(\mathrm{mm})$} \\
\hline$<5$ & 189 (61.0) \\
\hline $5-9.99$ & $108(34.8)$ \\
\hline$\geq 10$ & $13(4.2)$ \\
\hline
\end{tabular}

SD, standard deviation; SCC Ag, squamous cell carcinoma associated antigen; ASC, adenosquamous cell carcinoma; LVI, lymphovascular invasion; LN, lymph node; PALN, para-aortic LN.

Patients' characteristics are shown in Table 1.

\section{Evaluation of lymph node status}

The LN status was evaluated mainly by MRI. The LN status was assessed using a combination of size, shape, and internal architecture $[16,17]$. For determining the LN size, the short axis diameter of the largest LN was measured. At our institution, positron emission tomography/computed tomography (PET/ CT) has been in use since 2005. For evaluating a metastatic LN, 
PET/CT shows better accuracy than MRI. However, it cannot change the prognosis [18]. Therefore, we use mainly MRI rather than PET/CT for evaluating LN status.

\section{Treatment}

All patients were scheduled to receive combined external-beam radiotherapy (EBRT) and intracavitary brachytherapy (ICBT). Seventy-two patients received extended-field pelvic radiotherapy (EF-PRT), and the superior border was extended to encompass the PALN area. In the patients with PALN involvement $(n=17)$, PALN irradiation was done. In patients with no evidence of PALN involvement $(\mathrm{n}=55)$, the decision to use EF-PRT was at the discretion of the radiation oncologist, balancing the risk of occult PALN metastases against the potential for increased acute and late toxicity. In EF-PRT, the superior border was extended to encompass PALN area according to the discretion of the radiation oncologist as follows: T12-L1 ( $\mathrm{n}=20), \mathrm{L} 1-\mathrm{L} 2(\mathrm{n}=5)$, or L2-L3 $(\mathrm{n}=47)$ interspace. All patients received a median EBRT dose of $45 \mathrm{~Gy}$ (range, 39.6-54 Gy) at 1.7 Gy (in some EF-PRT cases only) or $1.8 \mathrm{~Gy}$ per fraction with whole pelvic radiotherapy (WPRT) or EF-PRT. After WPRT or EF-PRT, the boost irradiation of median $9 \mathrm{~Gy}$ (range, 5.4-23.4 Gy) given at 1.8 Gy or $2 \mathrm{~Gy}$ per fraction to $\mathrm{LN}$ regions that had significant evidence of carcinoma involvement or LN more than $10 \mathrm{~mm}$ on MRI findings, involved parametrium, or involved regions of the pelvic sidewall. In boost irradiation, three-dimensional conformal radiotherapy or intensity-modulated radiotherapy (IMRT) has been used since 2009. After adequate tumor regression, high-dose-rate ICBT was performed twice per week using an iridium-192 remote after-loading technique. The standard prescribed dose for each brachytherapy in our institution was 5.0 Gy to A-point in six fractions, twice weekly. The prescribed A-point dose was median $30 \mathrm{~Gy}$ (range, 15-36 Gy). The combined total dose from EBRT and ICBT was calculated using a linear quadratic model to determine the radiobiological equivalent dose in 2 Gy fractions (EQD2) $(\alpha / \beta=10)$ [19]. The median total prescribed EBRT EQD2 to pelvic LNs area and PALN area was 53.1 Gy (range, 44.25-69.03 Gy) and 44.25 Gy (range, 40.71-58.41 Gy). The median total prescribed A-point EQD2 (EBRT+ICBT) was 81.75 Gy (range, 69.36-105.70 Gy). The median overall irradiated time was 59 days (range, 45-133 days; interquartile range, 54-63 days).

All patients received radiotherapy and concurrent cisplatinbased chemotherapy. During radiotherapy, chemotherapy with weekly cisplatin ( $40 \mathrm{mg} / \mathrm{m}^{2}$ weekly for 6 weeks) was given to 200 patients. Two cycles of cisplatin-based combination chemotherapy with cisplatin plus 5-fluorouracil (5-FU), or cisplatin plus paclitaxel at 3 weeks intervals during external beam radiotherapy were given to 52 and 58 patients, respectively. Chemotherapy with cisplatin and 5-FU consisted of an intravenous infusion of $75 \mathrm{mg} / \mathrm{m}^{2}$ of cisplatin (day 1), followed by an intravenous infusion of $4,000 \mathrm{mg} /$ $\mathrm{m}^{2}$ of 5-FU over a 96-hour period (days 2-5). One liter of normal saline was given before and after cisplatin, and mannitol was used to increase the urine output (day 1). Chemotherapy with cisplatin plus paclitaxel consisted of an intravenous infusion of $135 \mathrm{mg} /$ $\mathrm{m}^{2}$ of paclitaxel (day 1), followed by an intravenous infusion of 75 $\mathrm{mg} / \mathrm{m}^{2}$ of cisplatin (day 2 ).

\section{Response evaluation and follow-up}

All patients were subjected to routine post-CCRT surveillance with physical examination, cervicovaginal cytology, laboratory test (e.g., squamous cell carcinoma antigen), and imaging studies, including abdominopelvic CT, MRI, and PET/CT. After completion of CCRT, the patients were evaluated every 3 months for the first 2 years and every 6 months thereafter. Recurrence was diagnosed through physical examination and diagnostic imaging (contrastenhanced CT, MRI, and/or PET/CT scans) $[16,17]$ and was confirmed histologically via needle aspiration or excisional biopsy when possible.

\section{End points and statistical methods}

The primary endpoint was in-field LN failure rate according to the size of LNs, and the overall survival (OS) rate and disease-free survival (DFS) rate according to the size of LNs. LN failure within the irradiated region was considered an in-field LN failure. We calculated all occurrences from the date of diagnosis to the date of relapse or the last date of follow-up. Deaths from other cause were censored at the time of last follow-up.

Comparison of variables was based on the t-test. The survival analysis was based on the life-table method of Kaplan-Meier. Univariate analyses were performed with log-rank tests. The Cox proportional hazard model was used to construct a multivariate model to predict survival. $p$-values were the result of two-sided tests and $p$-value $<0.05$ was considered statistically significant. Statistical analysis was performed using SPSS version 18.0 (SPSS Inc., Chicago, IL, USA).

\section{Results}

\section{Analysis of lymph node size}

The total number of patients was 310 . Patients were divided into four groups according to pelvic LN status and size evaluated by MRI. Of these, 168 patients had LNs that had no significant evidence of carcinoma involvement on MRI, and had a short axis of less than $10 \mathrm{~mm}$ (group 0 ). The other 142 patients had LNs with 


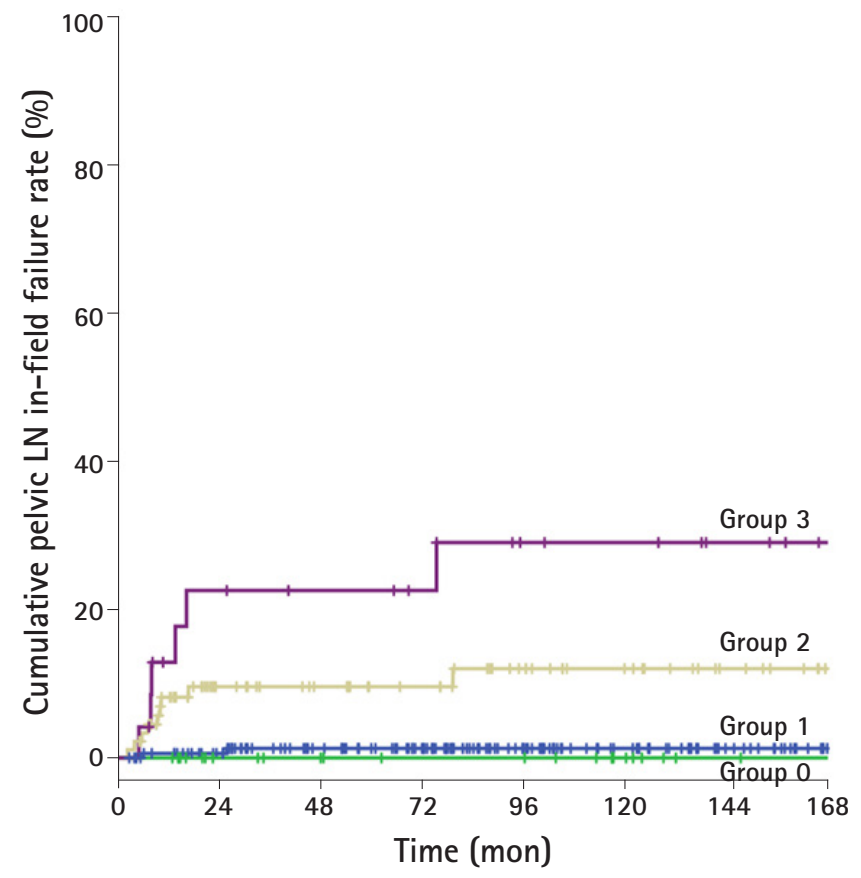

Fig. 1. Cumulative in-field pelvic $L N$ failure rate according to $L N$ size. LN, lymph node.

evidence of heavily involved carcinoma on MRI, and, these patients were further divided into three groups according to their LN size (group 1, <10 mm; group 2, 10-19.99 mm; group 3, $\geq 20 \mathrm{~mm}$ ).

On evaluation the in-field failure rate for the PALN area, 72 patients who received EF-PRT were analyzed. These patients were divided into three groups according to their PALN size (group 0, $<5 \mathrm{~mm}$; group 1, 5-9.99 mm; group 2, $\geq 10 \mathrm{~mm}$ ).

\section{In-field pelvic lymph node failure rate}

Due to the possibility of micro-metastasis of LN, the failure rate of all groups was analyzed. There was no significant difference between group 0 and group 1 (5-year in-field failure rate, 1.3\% and $0 \%$, respectively; 10 -year in-field failure rate, $1.3 \%$ and $0 \%$, respectively). The 5-year in-field failure rates among the patients in the groups 1,2 , and 3 were $0 \%, 9.6 \%$, and $22.6 \%$, respectively. The 10-year in-field failure rates among the patients in the groups 1,2 , and 3 were $0 \%, 12 \%$, and $29 \%$, respectively. There were statistically significant differences in the 5- and 10-year in-field failure rates between group 1 and group $2 / 3$ (group 1 vs. group 2, $p<0.001$; group 1 vs. group $3, p<0.001$ ). The in-field failure rate in patients with LN size $10 \mathrm{~mm}$ or more was significantly increased. In addition, although there was no statistically significant difference, the in-field failure rate tended to increase as the size increased (group 2 vs. group 3, $p=0.089$ ). The cumulative in-field pelvic $\mathrm{LN}$ failure rate according to $\mathrm{LN}$ size is shown in Fig. 1 .

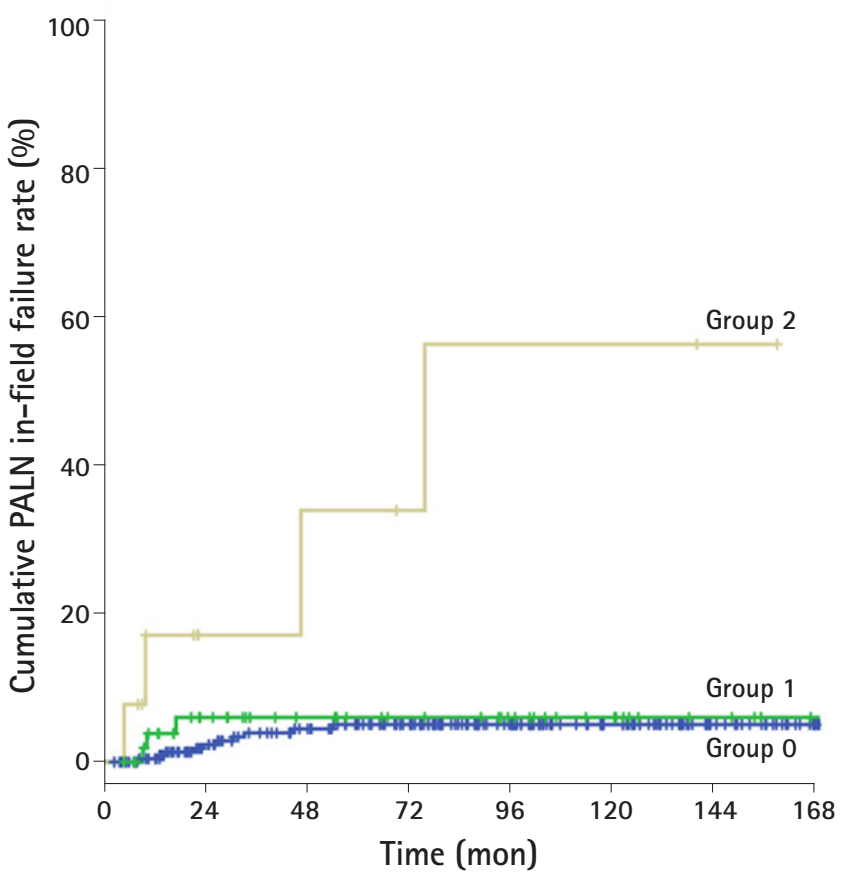

Fig. 2. Cumulative in-field PALN failure rate according to $L N$ size in patients treated with extended-field pelvic radiotherapy plus chemotherapy. PALN, para-aortic lymph node.

\section{In-field para-aortic lymph node failure rate}

The 5-year in-field failure rates among patients in groups 0,1 , and 2 were $5.9 \%, 5 \%$, and $18.5 \%$, respectively, and 10 -year in-field failure rates were $5.9 \%, 5 \%$, and $45.7 \%$, respectively. There were statistically differences between group $0 / 1$ and group 2 (group $0 / 1$ vs. group $2, p=0.024)$. Like the in-field failure rate for pelvic $\mathrm{LN}$, the in-field failure rate for PALN was significantly increased with LN size $10 \mathrm{~mm}$ or more. The cumulative in-field PALN failure rate according to $\mathrm{LN}$ size in patients treated with EF-PRT is shown in Fig. 2.

\section{Survival outcome: prognostic variables}

The results of the univariate analysis showed that advanced stage (I/II vs III/IV), pretreatment hemoglobin $(<12.3 \mathrm{~g} / \mathrm{dL})$, tumor size $(\geq 4 \mathrm{~cm})$, and $\mathrm{LN}$ size in the pelvic and para-aortic areas $(\geq 10$ $\mathrm{mm}$ ) were significant factors of poor OS rate and DFS rate. The 10 -year OS rate in patients with pelvic LN size $<10 \mathrm{~mm}$ and $\geq 10$ $\mathrm{mm}$ was $89.2 \%$ and $64.1 \%$, respectively, and the 10 -year OS rate in patients with PALN size $<10 \mathrm{~mm}$ and $\geq 10 \mathrm{~mm}$ was $82.4 \%$ and $33.3 \%$, respectively (Table 2 ). The OS rates were statistically different according to $\mathrm{LN}$ size $10 \mathrm{~mm}$ or more for both pelvic and PALNs (pelvic LN, $p<0.001$; PALN, $p<0.001$; Figs. 3 , 4). The 10 year DFS rate in patients with pelvic LN size $<10 \mathrm{~mm}$ and $\geq 10$ $\mathrm{mm}$ was $83.3 \%$ and $57.3 \%$, respectively, and the 10 -year DFS rate in patients with PALN size $<10 \mathrm{~mm}$ and $\geq 10 \mathrm{~mm}$ was $76.0 \%$ 
Table 2. Univariate survival analysis

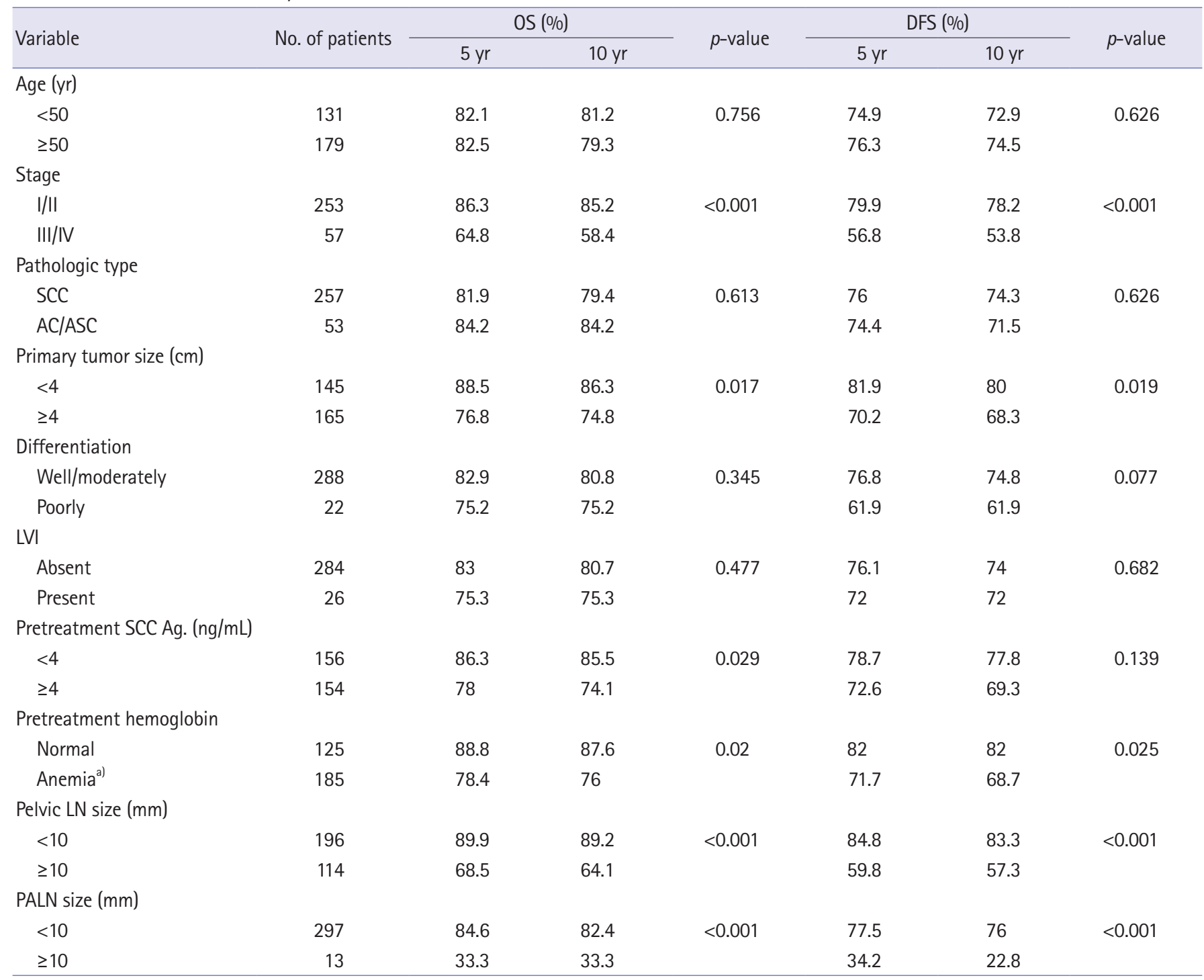

OS, overall survival rate; DFS, disease-free survival rate SCC, squamous cell carcinoma; AC, adenocarcinoma; ASC, adenosquamous cell carcinoma; LVI, lymphovascular invasion; SCC Ag, squamous cell carcinoma associated antigen; LN, lymph node; PALN, para-aortic lymph node.

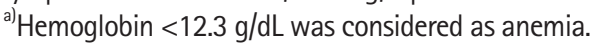

and 22.8\%, respectively. Of these, pelvic and PALN size $(\geq 10$ $\mathrm{mm})$ was a significant prognostic factor of OS and DFS rates in multivariate analysis (pelvic LN, $p=0.003$; PALN, $p=0.033$, Table 3). The irradiated dose was not significantly associated with poor OS and DFS rates.

\section{Discussion}

Although standard radiotherapy regimens have been established for the treatment of primary cervical cancer, optimal radiotherapy regimens for regional $\mathrm{LN}$ metastases remain unclear, particularly for bulky $\mathrm{LN}[20,21]$. The relationship between the size of metastatic LN and LN control in CCRT remains to be fully elucidated. In our study, LN size less than $10 \mathrm{~mm}$ was well-controlled, and the in-field failure rate for $\mathrm{LN}$ sizes $\geq 10 \mathrm{~mm}$ was increased. The in-field failure rate tended to increase as the $\mathrm{LN}$ size increased.

In the era of radiotherapy alone for advanced cervical cancer, Hacker et al. reported that the surgical removal of enlarged LNs prior to radiotherapy improves prognosis [22]. After the introduction of CCRT, since chemotherapy acts as a radiosensitizer, it may affect the control rate after bulky LN dissection, however, the results are insufficient. Lai et al. reported a study that assessed the prognostic significance of surgical staging in locally advanced cervical cancer [23]. The progression-free survival and OS rates of patients who underwent surgical staging were significantly poorer than those of patients who underwent clinical staging. It was suggested that the 


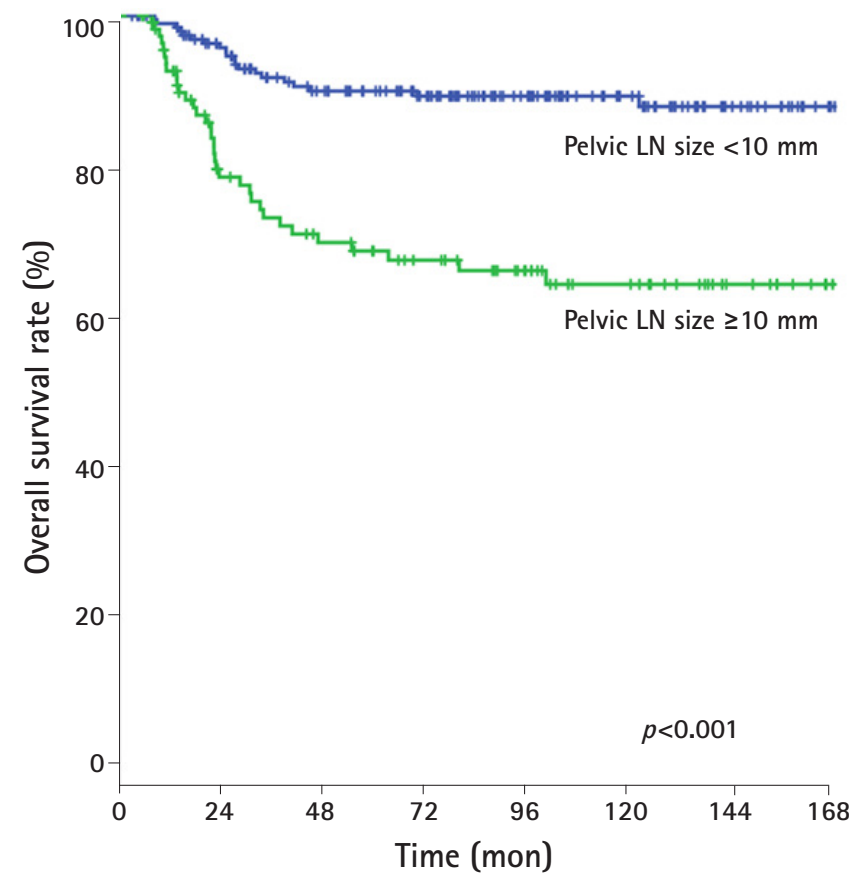

Fig. 3. Overall survival difference according to pelvic LN size. LN, lymph node.

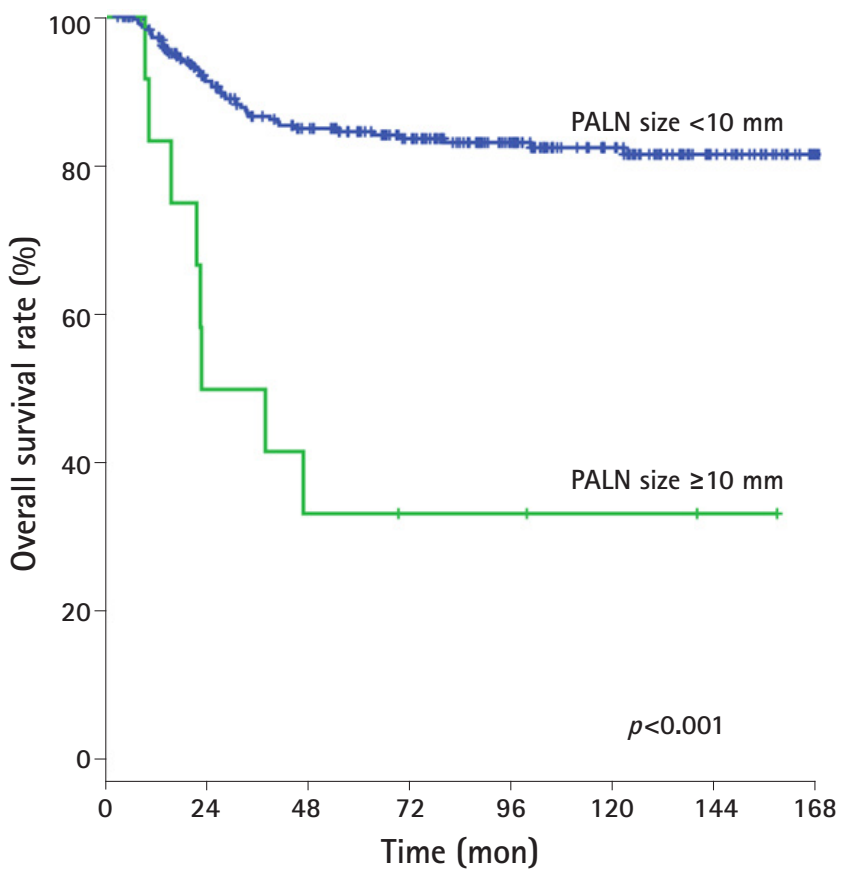

Fig. 4. Overall survival difference according to PALN size. PALN para-aortic lymph node.

Table 3. Multivariate survival analysis

\begin{tabular}{llll}
\hline Variable & RR & $95 \%$ Cl of RR & $p$-value \\
\hline Age & 1.011 & $0.983-1.039$ & 0.456 \\
Stage (I/II vs. III/M) & 0.55 & $0.267-1.132$ & 0.105 \\
Pathologic type (SCC vs. AC/ASC) & 0.991 & $0.418-2.351$ & 0.984 \\
Primary tumor size & 1.002 & $0.982-1.021$ & 0.874 \\
Differentiation (well/moderately vs. poorly) & 0.563 & $0.197-1.609$ & 0.283 \\
LVI (present vs. absent) & 0.872 & $0.349-2.178$ & 0.769 \\
Pretreatment SCC Ag. $(<4$ vs. $\geq 4 \mathrm{ng} / \mathrm{mL}$ ) & 1.01 & $0.999-1.021$ & 0.087 \\
Pretreatment hemoglobin (normal vs. anemia) & 0.994 & $0.838-1.179$ & 0.946 \\
Pelvic LN size $(<10 \mathrm{~mm}$ vs. $\geq 10 \mathrm{~mm})$ & 0.392 & $0.210-0.731$ & 0.003 \\
PALN size $(<10 \mathrm{~mm}$ vs. $\geq 10 \mathrm{~mm})$ & 0.402 & $0.174-0.927$ & 0.033 \\
\hline
\end{tabular}

$\mathrm{RR}$, relative risk; $\mathrm{Cl}$, confidence interval; SCC, squamous cell carcinoma; $\mathrm{AC}$, adenocarcinoma; $\mathrm{ASC}$, adenosquamous cell carcinoma; LVI, lymphovascular invasion; SCC Ag, squamous cell carcinoma associated antigen; LN, lymph node; PALN, para-aortic lymph node.

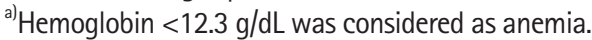

surgical assessment of PALN status prior to CCRT was ineffective compared with the use of imaging techniques [23-25]. Because the benefits of surgical dissection and biopsy are unclear, in the present clinical setting, oncologists use a radiologic method for the evaluation of LN status in almost all cases of locally advanced cervical cancer.

Therefore, currently, it is universally accepted that CCRT be performed following imaging studies in patients with locally advanced cervical cancer. The preoperative PET/CT evaluation of LNs assists in identifying distant metastasis and PALN metastasis, but dose not appear to improve survival rate. On evaluation of LN metastasis assessed by MRI, a wide range of sensitivity, specificity, and accuracy were reported; $30-73 \%, 93 \%$, and $73 \%$, respectively $[26,27]$. In addition, it has been suggested that the probability of microscopic LN disease is $20-25 \%$, even with no significant evidence of carcinoma involvement in an imaging study [28]. However, in our study, the in-field failure rate and OS differed only according to the LN size despite the limitation of clinical staging. For pelvic LNs, there was no significant difference between group 0 and group 1 (5-year in-field failure rate, 1.3\% and $0 \%$, respectively; 10 -year in-field failure rate, $1.3 \%$ and $0 \%$, respectively). Therefore, for an $\mathrm{LN}$ size of less than $10 \mathrm{~mm}$, the 
rate of control is similar regardless of whether the MRI reveals have significant evidence of carcinoma involvement. Negative LNs that had no significant evidence of carcinoma involvement or LNs less than $10 \mathrm{~mm}$ in MRI findings showed good control over conventional radiation dose, regardless of the sensitivity. Not all enlarged LNs are metastatic; however, they have a high metastatic potential. In our study, the factor of $L N$ size $\geq 10 \mathrm{~mm}$ was associated with an increase in the in-field failure rate and affected the survival outcome confirmed by multivariate analysis. Therefore, it is appropriate to use $\mathrm{LN}$ size measurement MRI in treatment planning.

There are several studies assessing the effects of LN size on prognosis. Studies have reported that an enlarged LN, based on 20 $\mathrm{mm}$ or $15 \mathrm{~mm}$ size, is associated with a poor prognosis $[15,29,30]$. Song et al. reported that the OS and DFS rate were poorer when the LN size was larger than $1.5 \mathrm{~cm}$ [15]. Since the study included mixed groups composed of patients treated with CCRT or radiotherapy alone, it is inadequate to validate the effect of CCRT. In the present study, the size of $10 \mathrm{~mm}$, which is suspicious of imaging positive lymphadenopathy, was used as the most commonly used size criteria. As a result, it was confirmed that an $L N$ size $\geq 10 \mathrm{~mm}$ affects the in-field failure rate and OS rate in a CCRT setting [31]. There was no significant difference in the in-field failure rate $(p=0.068)$ or OS ( $p=0.525$ ) when a pelvic $\mathrm{LN} \geq 20 \mathrm{~mm}$ was compared to a pelvic $L N$ size $\geq 10 \mathrm{~mm}$. Therefore, it is reasonable to use $10 \mathrm{~mm}$ as a criterion for determining positive $\mathrm{LN}$ status.

Optimal treatment with CCRT for an enlarged LN in locally advanced cervical cancer remains controversial. Traditionally, doses of 45-50 Gy in conventional fractionation are delivered to the whole pelvis to treat cervical cancer with or without concurrent chemotherapy, primarily due to adjacent normal tissue tolerance as a limiting factor. Subsequent small field radiation boosts of 5.4-10 Gy in conventional fractionation are frequently administered to metastatic LNs. The interdigitation pelvic node boosts with brachytherapy can present with specific challenges. An anteroposterior-posteroanterior boost technique may be used if boost fields are small and if less than an additional 5.4-10 Gy is needed to increase the combined external beam and brachytherapy dose to a minimum of $60-66$ Gy $[20,21]$. According to Toita et al., the prognosis was no poorer when the $\mathrm{LN}$ was irradiated with a dose less than that given using the conventional method [32]. Recently, Ariga et al. demonstrated that external beam boost irradiation to positive pelvic LNs achieves favorable nodal control without increasing late complications [13]. Hata et al. also reported the radiotherapy effectively controlled pelvic LN metastases in patients with cervical cancer with most $\mathrm{LNs}<24 \mathrm{~mm}$ in diameter controlled by the total dose of $50.4 \mathrm{~Gy}$ in $1.8 \mathrm{~Gy}$ fractions and radiation boost over 50.4 Gy may improve the control of metastatic LNs $\geq 24 \mathrm{~mm}$, particularly with concurrent chemotherapy [12]. They also suggested that higher doses to metastatic LNs may increase intestinal toxicities. In this CCRT study, the median total prescribed EBRT EQD2 to pelvic LNs area and PALN area was 53.1 Gy (range, 44.25-69.03 Gy) and 44.25 Gy (range, 40.71-58.41 Gy). It considered that in our study, pelvic LNs and PALNs that had evidence of heavily involved with carcinoma on MRI was given the dose as a traditional standard with concurrent chemotherapy. Despite the traditional standard dose in CCRT, our study showed that significantly higher incidence of in-field LN failure LNs recurrence in patients with pelvic $\mathrm{LN}$ size $\geq 10 \mathrm{~mm}$, than that in patients with pelvic $\mathrm{LN}$ size $<10 \mathrm{~mm}$. Therefore, the development of more effective radiotherapy strategies is required to reduce the pelvic $L N$ recurrence in patients with pelvic $L N$ size $\geq 10 \mathrm{~mm}$.

A higher dose than the traditional standard dose can be delivered using additional boost technique. Hata et al. reported that larger LNs that were $>24 \mathrm{~mm}$ in diameter may require higher doses, up to about $55.8 \mathrm{~Gy}$ [12]. Rash et al. reported that the control rate was improved when a total dose of $\geq 54$ Gy was delivered using a boost technique to treat pelvic and para-aortic lymphadenopathy [33]. However, results on the toxicity associated with higher radiation doses are insufficient. Normal tissue complication probability should be considered when increasing the radiation dose for achieving control of an enlarged LN. High-dose boost irradiation to enlarged LNs may increase the risk of high-dose exposure to the colon and small intestine due to their proximity to pelvic LNs. When higher boost doses are required, more complex techniques are recommended; however, to avoid compromising subsequent brachytherapy, care must be taken to minimize the dose to the bowel, rectum, and bladder from high-precision radiotherapy such as image-guided radiotherapy (IGRT) and IMRT. Recently, dose escalation studies have been performed using IMRT, volumetric modulated arc therapy, and IGRT; however, the problem of bowel toxicity remains, which limits the use of higher irradiation doses [34-36]. There are problems with the IMRT itself which are related to target definition, inter- and intra-fraction motion, and tumor regression during treatment $[37,38]$. However, some studies have shown that excellent control of the metastatic LNs with a median dose of 62 Gy (range, 59.4-64 Gy) using IMRT was achieved. Thus, we need to wait for the results of further randomized prospective trials and the result of long-term followup studies. Additionally, the use of high-precision radiotherapy such as image-guided stereotactic body radiotherapy or particle radiotherapy is expected to be beneficial for boost irradiation to enlarged LNs. By using these recent advanced treatment methods, higher doses can be delivered to the tumor without increasing 
doses to adjacent normal tissue.

A limitation of the present study includes its retrospective study design, which may be affected by selection bias. The LN dose by ICBT was not analyzed as image-guided brachytherapy was not performed, and irradiation dose to pelvic LNs may have been underestimated. There was a relatively small number of patients with PALNs of $\geq 10 \mathrm{~mm}$. Therefore, caution is required when ascribing clinical meaning to the results of the present study. The results of the present study must be validated on a larger patient cohort and further prospective randomized investigations of radiation dose escalation with IMRT are required to decrease pelvic LN recurrence.

In conclusion, the present study demonstrates that an LN of less than $10 \mathrm{~mm}$ in size in an imaging study was controlled by CCRT. On the other hand, in CCRT with boost irradiation to LNs of size $\geq 10 \mathrm{~mm}$, the in-field failure rate increases, and the prognosis deteriorates. Currently, treatment guidelines for enlarged LNs remain unclear; therefore, a more aggressive treatment strategy to overcome the adverse effects of enlarged LNs on survival outcomes is required.

\section{Acknowledgements}

This work was supported by research grants from the Catholic University of Daegu in 2017.

\section{Conflicts of interest}

No potential conflicts of interest relevant to this article was reported.

\section{ORCID}

Jinju Oh, https://orcid.org/0000-0003-1090-7637

Ki Ho Seol, https://orcid.org/0000-0001-6206-6425

Youn Seok Choi, https://orcid.org/0000-0001-8901-9434

Jeong Won Lee, https://orcid.org/0000-0001-7685-203X

Jin Young Bae, https://orcid.org/0000-0002-9287-8210

\section{References}

1. Lim MC, Lee M, Shim SH, Nam EJ, Lee JY, Kim HJ, et al. Practice guidelines for management of cervical cancer in Korea: a Korean Society of Gynecologic Oncology Consensus Statement. J Gynecol Oncol 2017;28:e22.

2. Eifel PJ, Winter K, Morris M, Levenback C, Grigsby PW, Cooper $\mathrm{J}$, et al. Pelvic irradiation with concurrent chemotherapy versus pelvic and para-aortic irradiation for high-risk cervical cancer: an update of radiation therapy oncology group trial (RTOG) 90-01. J Clin Oncol 2004;22:872-80.

3. Morris M, Eifel PJ, Lu J, Grigsby PW, Levenback C, Stevens RE, et al. Pelvic radiation with concurrent chemotherapy compared with pelvic and para-aortic radiation for high-risk cervical cancer. N Engl J Med 1999;340:1137-43.

4. Rossi PJ, Horowitz IR, Johnstone PA, Jani AB. Lymphadenectomy for patients with cervical cancer: is it of value? J Surg Oncol 2009;100:404-6.

5. Marana HR, de Andrade JM, Dos Reis FJ, Tiezzi DG, Zola FE, Clagnan WS, et al. Impact of surgical staging in locally advanced cervical cancer and subsequent chemotherapy. J Surg Oncol 2009; 100:505-10.

6. Goff BA, Muntz HG, Paley PJ, Tamimi HK, Koh WJ, Greer BE. Impact of surgical staging in women with locally advanced cervical cancer. Gynecol Oncol 1999;74:436-42.

7. Bae HS, Kim YJ, Lim MC, Seo SS, Park SY, Kang S, et al. Predictors of radiation field failure after definitive chemoradiation in patients with locally advanced cervical cancer. Int J Gynecol Cancer 2016;26:737-42.

8. Pilleron JP, Durand JC, Hamelin JP. Prognostic value of node metastasis in cancer of the uterine cervix. Am J Obstet Gynecol 1974;119:458-62.

9. Brenner DJ. Dose, volume, and tumor-control predictions in radiotherapy. Int J Radiat Oncol Biol Phys 1993;26:171-9.

10. Perez CA, Grigsby PW, Chao KS, Mutch DG, Lockett MA. Tumor size, irradiation dose, and long-term outcome of carcinoma of uterine cervix. Int J Radiat Oncol Biol Phys 1998;41:307-17.

11. Tanderup K, Fokdal LU, Sturdza A, Haie-Meder C, Mazeron $\mathrm{R}$, van Limbergen E, et al. Effect of tumor dose, volume and overall treatment time on local control after radiochemotherapy including MRI guided brachytherapy of locally advanced cervical cancer. Radiother Oncol 2016;120:441-6.

12. Hata M, Koike I, Miyagi E, Numazaki R, Asai-Sato M, Kasuya T, et al. Radiation therapy for pelvic lymph node metastasis from uterine cervical cancer. Gynecol Oncol 2013;131:99-102.

13. Ariga T, Toita T, Kasuya G, Nagai Y, Inamine M, Kudaka W, et al. External beam boost irradiation for clinically positive pelvic nodes in patients with uterine cervical cancer. J Radiat Res 2013;54:690-6.

14. Wakatsuki M, Ohno T, Kato S, Ando K, Noda SE, Kiyohara H, et al. Impact of boost irradiation on pelvic lymph node control in patients with cervical cancer. J Radiat Res 2014;55:139-45.

15. Song S, Kim JY, Kim YJ, Yoo HJ, Kim SH, Kim SK, et al. The size of the metastatic lymph node is an independent prognostic 
factor for the patients with cervical cancer treated by definitive radiotherapy. Radiother Oncol 2013;108:168-73.

16. McMahon CJ, Rofsky NM, Pedrosa I. Lymphatic metastases from pelvic tumors: anatomic classification, characterization, and staging. Radiology 2010;254:31-46.

17. Lai G, Rockall AG. Lymph node imaging in gynecologic malignancy. Semin Ultrasound CT MR 2010;31:363-76.

18. Tsai CS, Lai CH, Chang TC, Yen TC, Ng KK, Hsueh S, et al. A prospective randomized trial to study the impact of pretreatment FDG-PET for cervical cancer patients with MRI-detected positive pelvic but negative para-aortic lymphadenopathy. Int J Radiat Oncol Biol Phys 2010;76:477-84.

19. Joiner MC, Bentzen SM. Fractionation: the linear-quadratic approach. In: Joiner M, van der Kogel A, editors. Basic clinical radiobiology. 4th ed. London: Hodder Arnold; 2009. p. 102-19.

20. Viswanathan AN. Uterine cervix. In: Halperin EC, Wazer DE, Perez CA, Brady LW, editors. Perez and Brady's principles and practice of radiation oncology. 6th ed. Philadelphia: Wolters Kluwer Health/ Lippincott Williams \& Wilkins; 2013.p. 1355-425.

21. Eifel PJ, Klopp AH. Gynecologic radiation oncology: a practical guide. Philadelphia: Wolters Kluwer; 2017.p. 78-99.

22. Hacker NF, Wain GV, Nicklin JL. Resection of bulky positive lymph nodes in patients with cervical carcinoma. Int J Gynecol Cancer 1995;5:250-6.

23. Lai CH, Huang KG, Hong JH, Lee CL, Chou HH, Chang TC, et al. Randomized trial of surgical staging (extraperitoneal or laparoscopic) versus clinical staging in locally advanced cervical cancer. Gynecol Oncol 2003;89:160-7.

24. Brockbank E, Kokka F, Bryant A, Pomel C, Reynolds K. Pretreatment surgical para-aortic lymph node assessment in locally advanced cervical cancer. Cochrane Database Syst Rev 2013;(3):CD008217.

25. Vandeperre A, Van Limbergen E, Leunen K, Moerman P, Amant F, Vergote I. et al. Para-aortic lymph node metastases in locally advanced cervical cancer: Comparison between surgical staging and imaging. Gynecol Oncol 2015;138:299-303.

26. Bellomi M, Bonomo G, Landoni F, Villa G, Leon ME, Bocciolone L, et al. Accuracy of computed tomography and magnetic resonance imaging in the detection of lymph node involvement in cervix carcinoma. Eur Radiol 2005;15:2469-74.

27. Choi HJ, Roh JW, Seo SS, Lee S, Kim JY, Kim SK, et al. Comparison of the accuracy of magnetic resonance imaging and positron emission tomography/computed tomography in the presurgical detection of lymph node metastases in patients with uterine cervical carcinoma: a prospective study. Cancer 2006;106:914-22.

28. Gouy S, Morice P, Narducci F, Uzan C, Gilmore J, Kolesnikov-
Gauthier H, et al. Nodal-staging surgery for locally advanced cervical cancer in the era of PET. Lancet Oncol 2012;13:e212-20.

29. Inoue T, Chihara T, Morita K. The prognostic significance of the size of the largest nodes in metastatic carcinoma from the uterine cervix. Gynecol Oncol 1984;19:187-93.

30. Kodama J, Seki N, Ojima Y, Nakamura K, Hongo A, Hiramatsu Y. Prognostic factors in node-positive patients with stage IBIIB cervical cancer treated by radical hysterectomy and pelvic lymphadenectomy. Int J Gynaecol Obstet 2006;93:130-5.

31. Kim SH, Kim SC, Choi BI, Han MC. Uterine cervical carcinoma: evaluation of pelvic lymph node metastasis with MR imaging. Radiology 1994;190:807-11.

32. Toita T, Kitagawa R, Hamano T, Umayahara K, Hirashima Y, Aoki Y, et al. Phase II study of concurrent chemoradiotherapy with high-dose-rate intracavitary brachytherapy in patients with locally advanced uterine cervical cancer: efficacy and toxicity of a low cumulative radiation dose schedule. Gynecol Oncol 2012;126:211-6.

33. Rash DL, Lee YC, Kashefi A, Durbin-Johnson B, Mathai $\mathrm{M}$, Valicenti $\mathrm{R}$, et al. Clinical response of pelvic and paraaortic lymphadenopathy to a radiation boost in the definitive management of locally advanced cervical cancer. Int J Radiat Oncol Biol Phys 2013;87:317-22.

34. Simpson DR, Song WY, Moiseenko V, Rose BS, Yashar CM, Mundt AJ, et al. Normal tissue complication probability analysis of acute gastrointestinal toxicity in cervical cancer patients undergoing intensity modulated radiation therapy and concurrent cisplatin. Int J Radiat Oncol Biol Phys 2012;83:e81-6.

35. Verma J, Sulman EP, Jhingran A, Tucker SL, Rauch GM, Eifel PJ, et al. Dosimetric predictors of duodenal toxicity after intensity modulated radiation therapy for treatment of the paraaortic nodes in gynecologic cancer. Int J Radiat Oncol Biol Phys 2014;88:357-62.

36. Hegazy MW, Mahmood RI, Al-Badawi IA, Moftah B, AlHusaini H. Radiotherapy dose escalation with concurrent chemotherapy in locally advanced cervix cancer is feasible. Clin Transl Oncol 2016;18:58-64.

37. Collen C, Engels B, Duchateau M, Tournel K, De Ridder M, Bral S, et al. Volumetric imaging by megavoltage computed tomography for assessment of internal organ motion during radiotherapy for cervical cancer. Int J Radiat Oncol Biol Phys 2010;77:1590-5.

38. van de Bunt L, van der Heide UA, Ketelaars M, de Kort GA, Jürgenliemk-Schulz IM. Conventional, conformal, and intensitymodulated radiation therapy treatment planning of external beam radiotherapy for cervical cancer: the impact of tumor regression. Int J Radiat Oncol Biol Phys 2006;64:189-96. 